\title{
Multilevel Supporting System for Special Forms of Learning
}

\author{
${ }^{1}$ DALIBOR SLOVÁK, ${ }^{2}$ PETR LÁTAL \\ Computers and Communications System Department \\ Faculty of Applied Informatics Tomas Bata University Zlín, \\ CZECH REPUBLIC
}

\begin{abstract}
We created this system, because meanwhile another similar system does not exist. For the first usage and testing, multilevel supporting system for special forms of learning is being developed as a web-based support for teaching music theory. First part of the article is focused on basic description of the project. Main parts of multilevel supporting system for special forms of learning is described and described are using technologies for development of system too. In the next part of the article are described some CMS communications with publication and presentation. System is based on web technologies such as PHP, MySQL and HTML with fully featured java-script jQuery API and WYSIWYG editor TinyMCE with useful plugins. Last part is aimed to refer about all developed modules for the Multilevel supporting system for special forms of learning. We established the data-base structure of composers, music genres and music samples. It was necessary to create administration system where will be the teacher able to form the music staves and present them on the web portal. Everything is connected with the learning portal, which allows students to register, to apply for entry into the classes, receive the information, training and test their knowledge through the test module.
\end{abstract}

Keywords: Content management system, CMS Communications, Vexflow API, Cufon Content Management System Modules, Music stave generator, Quiz generator

Received: May 12, 2021. Revised: December 20, 2021. Accepted: January 17, 2022. Published: February 8, 2022.

\section{Introduction}

Multilevel supporting system for special forms of learning (in brief "MSFL") is being developed in the first instance as solution for teaching music theory in modern way. MSFL has features such as front-end layout for teaching and backend system for administration. System is written with web based technologies such as PHP, HTML, MySQL and contains some plugins such as TinyMCE WYSIWYG editor used for the easy work with document writing. We would like to describe in this paper all featured modules of the portal with fully outlined parts of each function. System is written with web based technologies such as PHP, HTML, MySQL and contains some plugins such as TinyMCE WYSIWYG editor used for the easy work with document writing. System has fully featured jQuery java-script library and special open-source API Vexflow which can display music staves.

This educational portal serves an environment for obtaining information in terms of fundamentals of music theory. The portal has the full-text search mechanism. Administrators can create any types of the tests that can be subsequently evaluated.

The aim of this work was to create a Web interface that would allow students to register on the portal, classify students into the training courses, create components for tests managing and develop the simple interface for teaching with many features connected with the each course.

\section{CMS}

\section{1 - Main features of CMS}

A content management system (CMS) is a computer system that allows publishing, editing, and modifying content as well as site maintenance from a central page. It provides a collection of procedures used to manage workflow in a collaborative environment. These procedures can be manual or computer-based.

The core features of Content Management Systems vary widely from system to system; many simpler systems showcase only a handful of features, while others, notably enterprise systems, are much more complex and powerful.

- Allow for a 1 arge number of people to share and contribute to stored data;

- Control access to data based on user role (i.e., define information users or user groups can view, edit, publish, etc.);

- Facilitate storage and retrieval of data;

- Control data validity and compliance;

- Reduce duplicate inputs;

- Simplify report writing;

- Improve communication among users.

Define data as almost anything: documents, movies, texts, pictures, phone numbers, articles etc.Finally, complete content and organizational editing before formatting. Please take note of the following items when proofreading spelling and grammar: 


\subsection{WCMS}

A Web Content Management System (WCMS) is a software system that provides website authoring, collaboration, and administration tools designed to allow users with little knowledge of web programming languages or markup languages to create and manage website content with relative ease. A robust WCMS provides the foundation for collaboration, offering users the ability to manage documents and output for multiple author editing and participation. Most systems use a Content Repository or a database to store page content, metadata, and other information assets that might be needed by the system.

A presentation layer displays the content to website visitors based on a set of templates. The templates are sometimes XSLT files.

Most systems use server side caching to improve performance. This works best when the WCMS is not changed often but visits happen regularly.

Administration is typically done through browser-based interfaces, but some systems require the use of a fat client.

A WCMS allows non-technical users to make changes to a website with little training. A WCMS typically requires a systems administrator and/or a web developer to set up and add features, but it is primarily a website maintenance tool for non-technical staff.

\subsection{Advanteges of CMS}

CMS is can be to create in WYSIWYG editors. You do not know HTML language, because you can use these editors for creating your web applications. Most of CMS systems are Open Source Software (OSS). It means, source codes are open for using and editing via Open source licenses.

CMS communication

Our CMS was developed to simplify the work as much as possible in creating of the new modules for communication between modules which have been secured against unauthorized access. Each page has been lightened by the administration of code that need not be loaded. The result is a system that communicates quickly and makes maximum use of optimized SQL queries.

Each module has its place on the ftp server in the /inc/ admin /. It is very important to have unique component containing as et of modifications (have to be named upravy.php) and a file containing error messages (have to be named hlasky.php). If you are missing these two files, you will be notified about this deficiency and asked to remedy it. It is good to have invented a meaningful file structure for adding, editing and general overview of the module. Finally is necessary to have super admin account that allows authorized user to use the "Settings Module". This module is important for adding or editing the new modules into the system and has special forms for security features, classify features and graphical features.

\subsection{Language Module}

The header file of language module is usually loaded once by system.php, which uses the php code filters for parsing country type and adds the value in the form of two lower case letter strings to the super global variable.(for example English has "en", Czech has "cs").

Then MySQL database queries the table "layout" and fetch data from the selected language to the website layout. If the language module cannot find a match with super global variable "lang", module will use the default language (in the case of teaching it is a Czech language). The database than fetches all data using the \$langcore["name_of the_cell"] syntax and then simply display the value of the cell by the PHP function echo. Front-end web interface has simple switcher for language changing.

\subsection{Content Creation}

Creating of the content in the MSFL system is very simple and efficient. This system in addition to their fundamental advantages such as creating and editing pages, deletion from the database, permitting or disabling content and other functions has also implemented multi-language support. In Figure 1 you can see the tree structure of the music portal website.

Layout of the website is designed for easy and quick user orientation in the content.

The greatest benefits in terms of content creation, is a simple environment that avail functions that allows users to work in similar way as in MS Word. MSFL has an integrated WYSIWYG TinyMCE plugin with predefined style Office 2007 with many plugins, which facilitate the working.

\subsection{Vexflow API}

VexFlow is the application programming interface for the music notation and can be used as a rendering engine for the various kinds of the online music learning tools, libraries, and applications. API is designed to run on HTML5 tag canvas with scalable vector graphics support.

It is important to note that the VexFlow is a low-level rendering API. Most applications wants to use something like VexTab which is a higher-level language for rendering guitar tablature and music notation.

VexFlow is written completely in JavaScript especially with jQuery API and using of HTML5 Canvas tag requires no external libraries or dependencies. For SVG support is necessary to include the Raphael JavaScript library to draw vector graphics on the MSFL site.

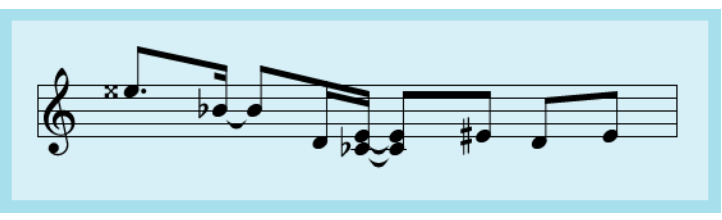

Fig. 1. Vexflow generated music stave element 


\section{Modules}

All modules are created exactly for learning portal and correspond with author's idea of music learning management system.

\subsection{Managing modules}

This is the most important module in the system. User should have to connect each module with the database and system very carefully. Fill in the wrong arguments could threaten the stability of the system. The main page of the module shows only active modules in the right corner, but you can see the all modules or only the active or inactive modules by choosing in select box. Each module has action module. These actions mean query from the administration to add, edit or view any module. Each action of the module has checked permissions in the database so any user cannot access, display or edit module without permissions.

\section{1) The procedure for creating new module}

Create folder for example: "testmodul" on the server in the directory /inc/admin/. Create files upravy.php, hlasky.php in this folder. Then we create files testadd.php for adding content, testedit.php for editing content and manage test.php for an overview. This created structure is needed to be linked with the system. Each module is quite complex so the new user does not immediately know how to fill form. For these purposes, each item is described and user should have problems with fill in necessary fields. For each module you can create "alias". This option was created for the easy administration of any tab. It gives possibility to add alias for any module in any tab fully associated with given module. These modules are marked with the star in modules manager.

\subsection{News module}

The module acts as a simple interface for adding new products to the main page of the site. It works with actions such as adding news, editing news and deletion of the news. It is important to choose the language equally as in the content module, name, dates and text news. The validation script will call you for the revalidation when you not complete all the boxes prompted. Date of news is facilitated by the simple jQuery calendar, which can easily offer current date listing of the months and years by clicking in the text area. The output data format is similar to the syntax of SQL function now (), so the work with date is very comfortable in system back-end.

\subsection{Users and rights}

Users of the system are an integral part of the distribution system management roles. Here you can find ordinary users who have been registered on the web site and users inserted to the system as the system administrators. Users are simply categorized in the group. Module contains tab with the current distribution of groups in database. Each group has assigned value. This value means that if the user has higher value, he has higher permissions with access. For example the group named super admin has value 3 so this user can access anywhere. Group named administrators have value 2 so this user cannot access to the modules management.

Authorized user can easily edit and assign different rights to another user. Module offer editing such as the password change and some changes of the user access and all the personal information.

\subsection{Photo gallery module}

Photo gallery module was created for fast adding of images and integration to the system. This module is connected to the module "folder administration," because of each image classification. If you do not create the folder tree, you can upload all the photos into one folder, but this may result in bad output on the web. Each photo is re-editable, erasable and can be edited directly on the server. Paging module is implicitly set to 30 images on the page, but this value can be changed in the system. Adding photos is a very simple and powered by link "Add photo". You just fill out the category name, description of photos and choose a photo from the local computer.

\subsection{Server image editor}

Image manager contents simple image editor. You can use the editor for set-up of images directly in database. E.g you can turn cut or change size of your images. You need not local image editor in your computer. When your change is over, you can save your image on the server. Image will be saved into two files. The first will be original of your picture and second file will be modified picture. You only see modified version of your picture, but after renaming of pre-editing original you can use this file as a real original file.

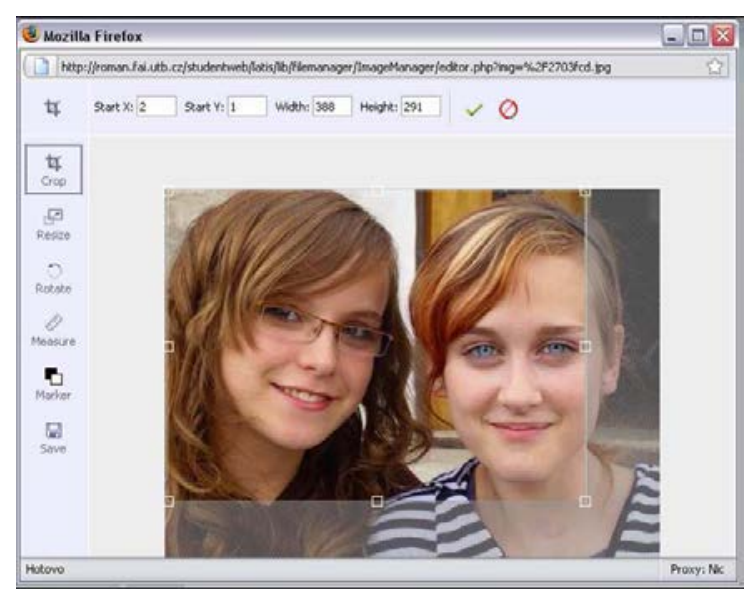

Fig. 3. Server Image Editor

\subsection{Folder administration modul}

This is a very strategic module of the system. Each part of the site is categorized in some way. System will upload photos, videos, or link different modules (eg "quiz module" or "course module") depending on the created folder structure. Folder administration database is the distribution of different 
types of web content. Adding of the new components is performed by the link "add folder" and again it is important to select the category to the new folder and select the language.

\subsection{Site settings}

This is essentially the language module that is used to modify the language simple layout via the web interface in administration. We are able to add a new cell to the database with properties that can then be applied anywhere on the web by the sophistication of this module. Items are categorized as the data type, date and name of the item. The item ID cell is displayed only and cannot be edited because of identifier which contains necessary information for modification in database.

\subsection{Courses management}

This module has similar module components to file management module, because you can create independent tree structure of courses. Each course contains a name, description of the course, password for the course and course leader. It is possible to set whether the course is active or not. In the list of courses is the list of registered students and the course leader. To see the all students named in the course, just click on the link named registered students. If you want to add student to the list, just add him using the "add user" link. In the point of course creation is necessary to select the right category, write the name of the course than create a password for the course and associate it with head of the course. It would be good to create a s hort description of the course, which will be displayed on the site and will serve as information for candidates. In the head of course is possible to choose registered users from select box. If the user does not exist, it is necessary to create him in the module "Users and rights" and then add him the appropriate permissions.

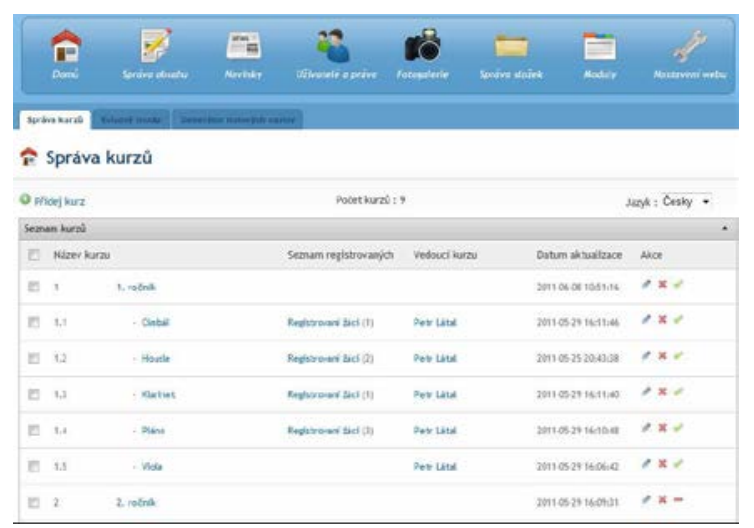

Fig. 4. Course management

\subsection{Sheet of music generator}

Module for generating staves is served as the simplified interface for creating musical staves for the quiz module and for the presentation of given expressions on the web. The main features of the module include the name of the stave, connection with the test and stave status.

From the database perspective the module contains a d ata section, which is generated by module and stored in a database as a complete code ready for presentation.

\subsection{Quiz module}

This is the module that generates any types of quiz or test. The basic properties of this module are the name of the quiz results, categories, and the basic operations such as editing, adding and deleting quizzes. For each quiz you can take a look at the results of every student who completed the quiz. There is a visible success, the date of attempt and the answers structure of the student.

\section{Conclusion}

The aim of this article was to describe the all features and modules of the MSFL. System has various kinds of modules connected together. Administrator can manage, create and edit all the contents of the portal, manage users and rights, quizzes and musical stave generator and many other modules. Administrator requires just a basic knowledge of music theory. There is required only the syntax for writing the music staves which can be connected with quiz module and presented on the web portal. Management is fully featured with the multilanguage administration, so administrators can easily manage any module in any language.

\section{References}

[1] Henk C.A : van TILBORG (2000). Fundamentals of cryptology, Kluwer Academic, ISBN 0-7923-8675-2, Norwell.

[2] Schlossnagle, Georgie (2004). PHP 5 advanced programming, Computer Press, ISBN 80-86815-14-5, Brno.

[3] Composite authors (2007), PHP 5 mastery, Computer Press, ISBN 97880-251-1519-0, Brno.

[4] Resig, John (2007). Javascript a AJAX : modern programming of web applications, Computer Press, ISBN 978-80-251-1824-5, Brno.

[5] Zelinka, Ivan. (1999). Applied informatics, Editorial center, UTB, ISBN 80-214-1423-5, Zlin

[6] Castro, Elizabeth (2007). HTML : XHTML a CSS: web site creating. Computer Press, ISBN 978-80-251-1531-2, Brno.

[7] RESIG, John. (2007) Javascript a AJAX : Moderní programování webových aplikací. Computer Press, ISBN 978-80-251-1824-5. Brno.

[8] Latal, P. Web portal for teaching music theory: Tomas Bata University, Faculty of Applied Informatics, Department of Computer and Communication Systems, 2011, 87 p. Thesis supervisor Ing. Dalibor Slovak.

[9] Ullman, Larry (2004). HTML : PHP a MySQL, Computer Press, ISBN 80-251-0063-4, Brno.

[10] Composite authors (2007), PHP 5, Computer Press, ISBN 978-80-2511519-0, Brno.

[11] Wexflow [available online]: $<$ http://vexflow.com/docs/tutorial.html $>$

[12] Cufonlibrary[available online]: $<$ https://github.com/sorccu/cufon/wiki/About $>$

[13] Raphael library [available online]: Cufon [available online]: $<$ http://raphaeljs.com/>

\section{Creative Commons Attribution License 4.0 (Attribution 4.0 International, CC BY 4.0)}

This article is published under the terms of the Creative Commons Attribution License 4.0 https://creativecommons.org/licenses/by/4.0/deed.en_US 